\title{
Optimization of piezoelectric ultrasound emitter transducers for underwater communications
}

\author{
M. Martins ${ }^{\mathrm{a}, *}$, V. Correia ${ }^{\mathrm{a}}$, J.M. Cabral ${ }^{\mathrm{a}}$, S. Lanceros-Mendez ${ }^{\mathrm{b}, \mathrm{c}}$, J.G. Rocha $^{\mathrm{a}}$ \\ a University of Minho, Algoritmi Research Center, Campus de Azurem, 4800-058 Guimaraes, Portugal \\ b University of Minho, Center/Department of Physics, Campus de Gualtar, 4710-057 Braga, Portugal \\ ' INL - International Iberian Nanotechnology Laboratory, 4715-330 Braga, Portugal
}

\section{A R T I C L E I N F O}

\section{Article history:}

Received 25 January 2012

Received in revised form 13 June 2012

Accepted 13 June 2012

Available online $\mathrm{xxx}$

\section{Keywords:}

Underwater acoustic channel

Underwater wireless communication

Transducer optimization

Piezoelectric ultrasound emitter

transducers

\begin{abstract}
A B S T R A C T
Ultrasound transducers are typically based on piezoelectric materials, due to their good response at high frequencies. Depending on the application, ceramics, polymers and composite materials can be used. In this work, an optimization study of ultrasound transducers for underwater communications is addressed, focusing on a piston type emitter transducer operating in thickness mode $\left(d_{33}\right)$. The piston is constituted by an active element disk with optimized dimensions. It is discussed how the acoustic impedance, thickness, resonance frequency and structure affect the transducer performance. This work allows a better understanding of the emitter transducer characteristics allowing reaching the optimum point of operation for specific applications. Focusing on underwater communication, the acoustic channel is defined and the transducer is optimized by finite element computer simulations. The results were compared with experimental tests, which show that four-layer structures increase up to $16 \mathrm{~dB}$ in performance versus single-layer.
\end{abstract}

(c) 2012 Elsevier B.V. All rights reserved.

\section{Introduction}

The piezoelectric properties of some materials are one of the key issues for underwater sound communication developments, being most of the ultrasound transducers based on piezoelectric materials. Piezoelectric properties can be found in certain types of ceramics, polymers and composites, each one of them showing different characteristics in terms of acoustic impedance, stiffness, coupling coefficient, piezoelectric and dielectric response. There are two types of ultrasound transducers: projectors, which convert electrical energy into mechanical energy and hydrophones, which perform the opposite conversion [1,2].

Applications of ultrasound transducers range from underwater sonars [3-5] to medical imaging and biomedical applications [6]. In the case of sonars, the acoustic signal has low frequency and can reach several tens of kilometers. On the other hand, medical imaging works with higher frequencies, reaching hundreds of $\mathrm{MHz}$, with signals reaching just few centimeters. This technology shows an interesting and useful gap concerning underwater applications, as there are no specific transducers for wireless broadband communications [3-14].

This work is focused in projector type ultrasound transducers with piston architecture. The piston-type transducer generally

\footnotetext{
* Corresponding author. Tel.: +351932843895.

E-mail address: mmartins@dei.uminho.pt (M. Martins).
}

projects the sound into a directional beam, whose directionality depends on the relationship between its size and the wavelength of the ultrasound signal. In this study the piston is a piezoelectric plate, which operates in the thickness mode [2].

The main goal is to develop a transducer that works at frequencies up to $1 \mathrm{MHz}$, able to communicate at distances up to one hundred meters. These values were chosen considering underwater channel characteristics such as sound attenuation and propagation speed $[1,2]$.

\section{Background}

\subsection{Underwater acoustics channel}

The underwater acoustic channel has specific properties that must be taken into account in order to properly define specific transducers [2]. In this study the focus is on the propagation speed and attenuation, being among the most relevant parameters to be considered for underwater communication applications [7-14].

The propagation speed is required to calculate the propagation delay and defines the transducer dimensions. The attenuation helps to understand how the signal travels along the medium. In this way, the signal amplitude at the receptor can be predicted and the sound pressure amplitude in the projector adjusted in order to reach the desired distances. 


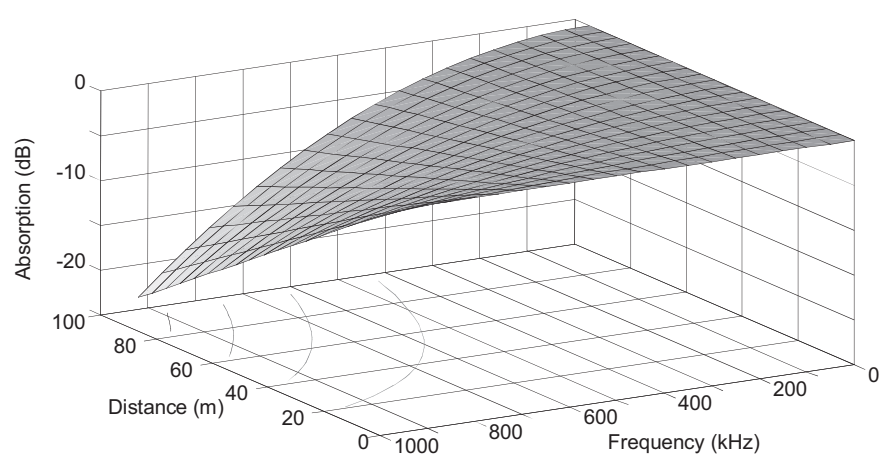

Fig. 1. Underwater acoustic channel absorption as function of frequency and distance.

The sound speed depends on the properties of the propagation medium (water). The dependence of sound speed on density $\rho$ and bulk modulus $B$ of the medium is given by:

$c=\sqrt{\frac{B}{\rho}}=\sqrt{\frac{1}{k \rho}}$

where $c$ is de sound speed and $k=1 / B$ in homogenous liquids and gases $[2,13]$. The sound speed in water is influenced by the temperature, salinity and depth such that increasing of any one of these parameters leads to an increase of sound speed by Eq. (A.2) [15]:

$c=1449.2+4.6 T-0.055 T^{2}+0.0029 T^{3}+(1.34-0.01 T)(S-35)$

$$
+0.06 D
$$

where $T$ is the water temperature, $S$ the salinity in parts per million, and $D$ is the depth.

Attenuation depends on two factors: distance and frequency, and is influenced by two major factors: spreading loss and absorption loss.

A simplified formula to describe the absorption coefficient as a function of frequency $(a(f))$ can be obtained from two major mechanisms: viscous absorption (above $100 \mathrm{kHz}$ ) and chemical relaxation (below $100 \mathrm{kHz}$ ). The last one is composed by boric acid (up to a few $\mathrm{kHz}$ ), and magnesium sulfate (up to $100 \mathrm{kHz}$ ). The absorption loss is given by Eq. (A.3) [16]:

$$
\begin{aligned}
a(f)= & \frac{0.106\left(f_{1} f^{2}\right) e^{(\mathrm{pH}-S / 0.56)}}{f_{1}^{2}+f^{2}}+\frac{0.52(1+T / 43)(S / 35)\left(f_{2} f^{2}\right) e^{(-D / 6)}}{f_{2}^{2}+f^{2}} \\
& +0.00049 f^{2} e^{-(T / 27+D / 17)} f_{1}=0.78 \sqrt{(S / 35)} e^{(T / 26)} \text { and } f_{2} \\
= & 42 e^{(T / 17)}
\end{aligned}
$$

where $\mathrm{pH}$ represents the $\mathrm{pH}$ of water and $f_{1}$ and $f_{2}$ are the relaxation frequencies introduced by boric acid and magnesium sulfate.

Fig. 1 shows a simulation of the absorption (Eq. (A.3)) in a subaquatic acoustic channel for frequencies up to $1 \mathrm{MHz}$ and distance up to $100 \mathrm{~m}$ in a fresh water medium with $15^{\circ} \mathrm{C}$ of temperature, $1 \mathrm{~m}$ of depth and $\mathrm{pH}$ of 7.

Fig. 1 shows how the sound absorption evolves with increasing frequency or distance.

The spreading loss results from the dispersion of the acoustic wave in the medium. In a homogenous and infinitely extended medium, the energy in the form of an acoustic pressure wave is radiated in a beam of sound with some specific angle. As the total area of the pressure wave surface increases with distance, the energy is spread for that area. The intensity of sound is equal to the pressure output power per unit area, decreasing therefore as the wave moves away from the source [2].

$I_{0}=\frac{P a}{A_{r 0}}, \quad I=\frac{P a}{A_{r}}$

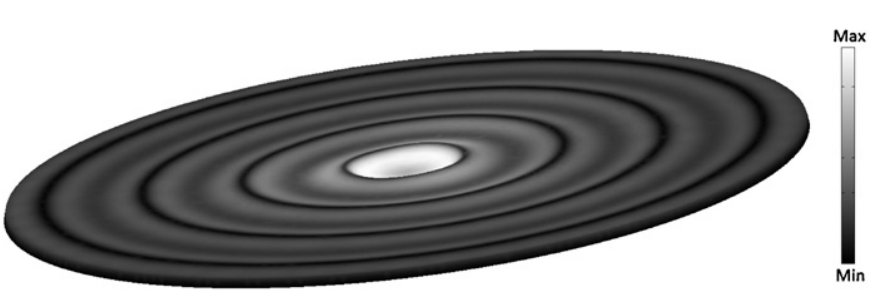

Fig. 2. 3D representation of the active element plate deformation when excited with a sine wave signal.

where $A_{r 0}$, is the area at reference distance $(1 \mathrm{~m}), A_{r}$ is the area at distance $r, P_{a}$ is the acoustic power source, $I_{0}$ is the acoustic intensity at distance $r_{0}$ and $I$ is the acoustic intensity at distance $r$.

In this way, it is possible to quantify the decrease in acoustic signal intensity resulting by signal spreading. The spreading loss $\mathrm{g}(\mathrm{r})$ is equal to:

$g(r)=10 \log _{10}\left[\frac{I_{0}}{I}\right]=10 \log _{10}\left[\frac{A_{r}}{A_{r 0}}\right]$

and therefore [8]:

$10 \log _{10} A(f, r)=g(r)+\frac{a(f) \cdot r}{1000}=10 \log _{10}\left[\frac{A_{r}}{A_{r 0}}\right]+\frac{a(f) \cdot r}{1000}$

In this way, the spreading loss just depends on distance and absorption loss just depends on frequency.

\subsection{Piezoelectric transducer considerations}

Piezoelectric materials are commonly used in the fabrication of ultrasound transducers, once they show very good response at high frequencies, which can reach up to $160 \mathrm{MHz}$ in the case of polymer materials [17].

Piezoelectric ultrasound transducers at high frequencies usually operate in the 33 mode, that is, the deformation along the polarization axis and the excitation electric field point into the same direction. The free displacement of the material in direction 3 , without restraining force and assuming uniform strain over the surface, is given by:

$\xi=d_{33} v$

where $\xi$ is the free displacement, $V$ is the applied voltage and $d_{33}$ is the coupling coefficient in the thickness direction. In most of the cases, the piezoelectric deformation is not homogenous and linear as it is typically assumed [1]. In the case of a piston transducer, the largest deformation occurs at the center of the plate and smoothes away from the center in an oscillation curve as shown in Fig. 2.

Fig. 2 is the finite element simulation result of a PZT-5H plate with $4 \mathrm{~mm}$ of diameter and $55 \mu \mathrm{m}$ of thickness, excited with a $10 \mathrm{~V}$ sine wave signal amplitude and a frequency of $10 \mathrm{MHz}$. The deformation amplitude is not relevant in this case. The important information from this simulation is how the active element deforms when excited with a sine wave signal. This wavy surface do not generate a homogeneous sound wave, therefore it is essential to consider this effect to simulate the transducer behavior.

The maximum force the piezoelectric element can apply to a medium is obtained by [1]:

$F=d_{33} Y_{3}^{E} \frac{A_{p}}{t_{p}} v, \quad S_{33}^{E}=\frac{1}{Y_{3}^{E}}$

where $S_{33}^{E}$ is the elastic compliance coefficient, $A_{p}$ is the area of the piezoelectric element and the $t_{p}$ is the thickness. The deformation creates a pressure wave in the medium, whose amplitude can be obtained by [2]:

$F=p A_{p}, \quad p=c \rho \pi f \xi$ 


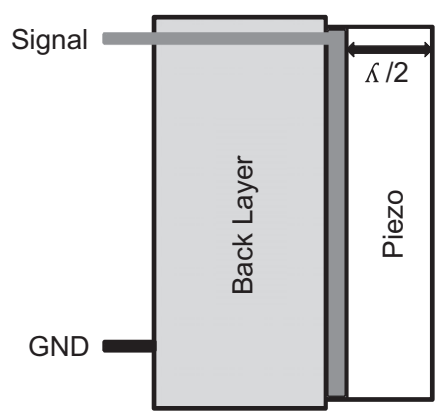

Fig. 3. 2D representation of a piston transducer.

where $c$ is sound speed, $\rho$ is the material density, $f$ is the signal wave frequency and $\xi$ is the piezoelectric material displacement. In order to increase pressure wave amplitude, several piezoelectric disks in a stack configuration can be used. This configuration allows multiplying the free displacement by the number of layers, $n$. In this case, the strain is given by [18]:

$\xi=d_{33} v n$

Despite this increase in strain, the intensity of the produced force does not change $[1,18,19]$.

Nevertheless, this approach reveals a problem: by increasing the number of layers, the electrical capacitance of the transducer also increases, which in turn represents a decrease of the imaginary part of the impedance and therefore an increase of the absolute value of the reactive electrical power.

To properly design ultrasound transducers it is important to consider the acoustic impedance of materials, in order to minimize energy losses due to acoustic impedance mismatches [2].

If the acoustic impedance mismatch is very high, the wave is highly reflected. Therefore, the transducer output must have a thickness equal to half wave, as it is shown in Fig. 3 [2]. In this way, the reflected wave will be synchronized with the next pressure wave cycle. The fundamental resonance frequency can be calculated from [2]:

$f_{r}=\frac{c}{2 t}$

where $t$ is the piezoelectric thickness and $c$ is the sound speed. The harmonics are constituted by odd and even resonance frequencies, given by:

$f_{\text {odd }}=(2 n+1) f_{r}, \quad n=0,1,2,3,4 \ldots \ldots$

$f_{\text {even }}=(2 n) f_{r}, \quad n=0,1,2,3,4 \ldots \ldots$

The back layer creates a boundary to reflect most of the pressure wave and to not allow the active element layer to deform in that direction. The signal electrode is placed in the interface between layers in order to reduce electromagnetic interferences by using a shield connected to the electrical ground [2].

The acoustic beam has a pattern characterized by its divergence angle, which depends on the transducer diameter and on the wavelength. The value of half beam divergence angle $\delta$ for a sound speed of $1500 \mathrm{~m} / \mathrm{s}$ (in water) is given by [2]:

$\delta=\arcsin \left(\frac{\lambda}{D}\right)$

\section{Selection of materials}

There are several piezoelectric materials available for ultrasound transducers. The most common are the Lead zirconate titanate (PZT), lead titanate (PT), lead magnesium niobate (PMN) and lead zinc niobate (PZN) ceramics and polyviylidenefluoride
Table 1

Comparison of some characteristics of PZT and PVDF.

\begin{tabular}{lll}
\hline Physical property & PZT-5H & PVDF \\
\hline Acoustic impedance $(Z)\left[10^{6} \mathrm{~kg} / \mathrm{m}^{2} \mathrm{~s}\right]$ & 33.15 & 2.7 \\
Resonance frequency $(f)[\mathrm{MHz}]$ & $<25$ & $<160$ \\
Piezoelectric coefficient $\left(d_{33}\right)\left[10^{-12} \mathrm{~m} / \mathrm{V}\right]$ & 512 & -34 \\
Relative dielectric constant $\left(\varepsilon_{\mathrm{r}}\right)$ & 3100 & 12 \\
Maximum operating temperature $\left[{ }^{\circ} \mathrm{C}\right]$ & 365 & 80 \\
\hline
\end{tabular}

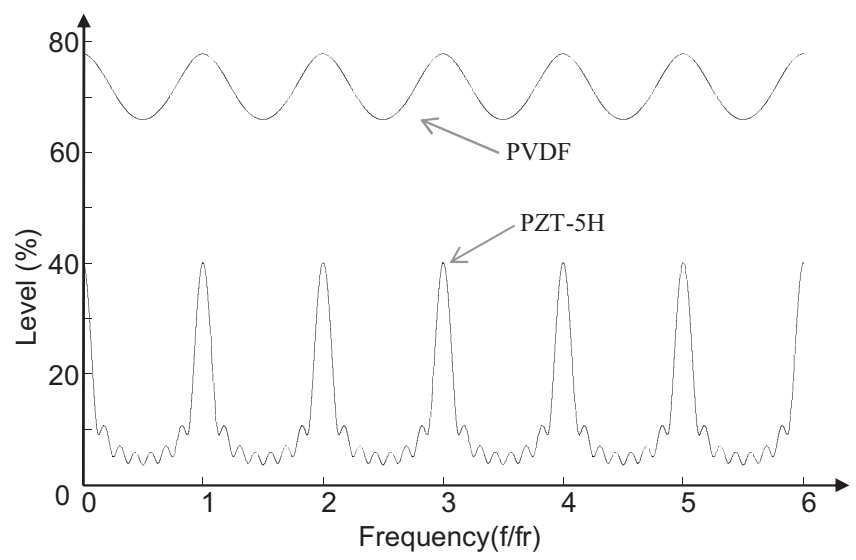

Fig. 4. PZT-5H and PVDF responses over 7 cycles, for a frequency $(f)$ range from 0 to 6 times the resonance frequency ( $f r)$.

(PVDF) and P(VDF-TrFE) polymers. Single crystals of PZT, PMN and PZN can also be used [17-30].

This study will focus on PZT-5H ceramic and PVDF polymer, since these are the most interesting materials for large scale applications due to their availability and price. The results will be nevertheless representative for their respective family of materials (ceramic and polymer piezoelectrics, respectively). Table 1 shows the main physical properties of these materials for the proposed application.

The resonance frequencies and the acoustic impedance are related one another. The sound pressure variation level with frequency depends directly on the acoustic impedance mismatch between the active element and the medium (water). Over signal cycles, the reflected sound wave may be added or not to the next cycle, thus increasing the output level. Eq. (A.15) gives the transmitted sound wave percentage over $n$ cycles.

$S_{\text {out }}=T_{w}\left(\sum_{i=0}^{n} R_{w}^{2 n} \times \sin \left(2 \pi f+n \times D_{p}\right)\right)$

$S_{\text {out }}$ is the sound wave output percentage over $n$ cycles, $T_{w}$ is the percentage transmitted sound wave intensity, $R_{w}$ is the percentage reflected sound wave intensity and $D_{p}$ is the delay of the reflected sound wave, introduced by the active element thickness. Table 1 shows the reflected and transmitted sound wave percentages in water (with a water acoustic impedance of approximated $1.5 \times 10^{6} \mathrm{~kg} / \mathrm{m}^{2} \mathrm{~s}$ ), for PZT and PVDF.

Fig. 4 is obtained by the incorporation of Table 2 values in to Eq. (A.15), which results in percentage level of the transmitted sound wave intensity to the medium over 7 cycles of a sine wave signal,

Table 2

Reflected and transmitted sound wave percentages in water, produced by PZT and PVDF.

\begin{tabular}{llc}
\hline Material & Reflected & Transmitted \\
\hline PZT-5H & $91.3 \%$ & $8.7 \%$ \\
PVDF & $28.7 \%$ & $71.3 \%$ \\
\hline
\end{tabular}




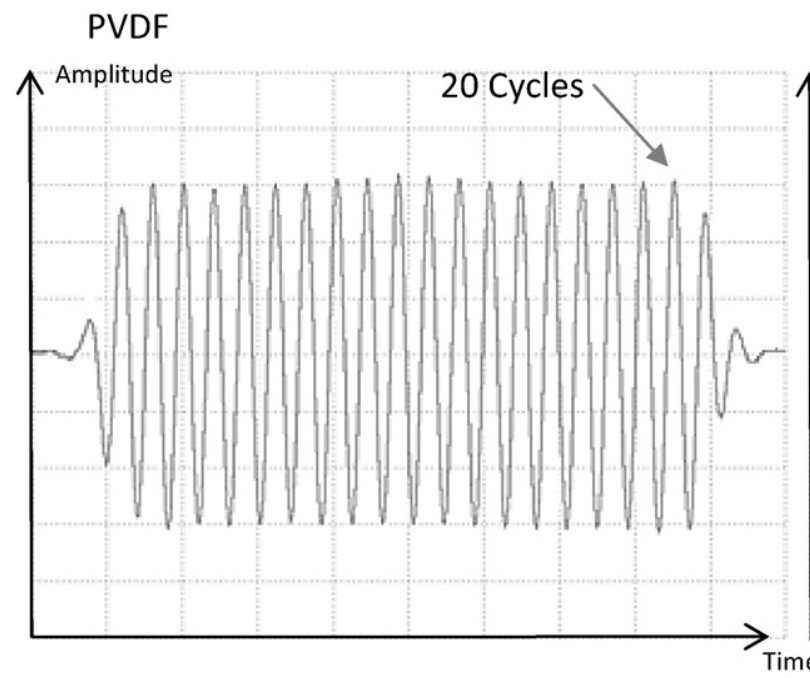

\section{PZT-5H}

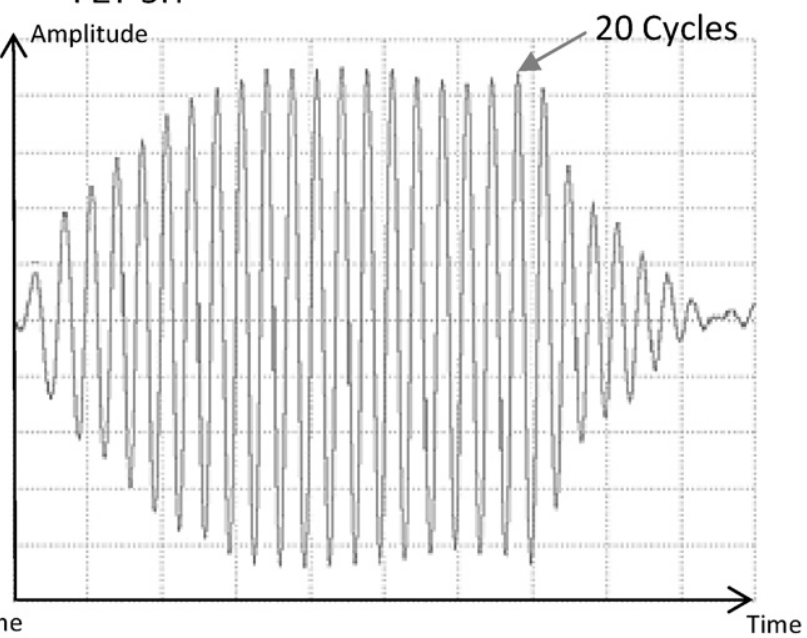

Fig. 5. Sound wave created by PVDF and PZT-5H transducer with $1 \mathrm{MHz}$ sine wave signal over 20 cycles.

as shown in Fig. 5, since after these 7 cycles the sound wave output intensity begins to stabilize. It is possible to observe the resonance, resonance harmonics and anti-resonance frequencies points from $0 \mathrm{~Hz}$ to 6 times the resonance frequency to a certain transducer thickness and material [2].

As it can be seen in Fig. 4, the percentage level of the sound power output over the frequencies shows a very low flotation to the PVDF, when compared with PZT-5H.

With the increasing of the reflected sound wave inside the transducer, increases the time necessary to reach the maximum output power at the resonance frequency and it is created a sound wave where the amplitude shows growth, therefore deforming the signal. The opposite effect happens when it is shut down the excitation signal to the transducer where the sound wave amplitude shows a decreasing; this oscillation is caused by the remaining sound wave power inside the transducer. Fig. 5 shows the sound wave created by a sine wave signal over 20 cycles of $1 \mathrm{MHz}$ signal using transducers of PVDF and PZT-5H with the same dimensions and in a resonance thickness.

As it is possible to observe in Fig. 5, the sound wave generated by the PVDF transducer has a more uniform signal than the sound wave generated by transducer PZT-5H. If we consider the results shown in Figs. 4 and 5, it can be concluded that, for a transducer operating in broad band of frequencies, PVDF shows more promising results. But in order to optimize other types of materials and reduce the output flotation, two solutions can be implemented. In the first solution the operating frequencies are restricted to integer multiples of the resonance frequency. The second solution it is basically a transducer designed for very $\operatorname{high} f_{r}$, which operates with frequencies much lower than $f_{r}$. The second solution will be used to compare PVDF and PZT performances. Fig. 6 shows the transmitted coefficient percentage level of the sound power for frequencies up to 0.1fr in PVDF and 0.05fr in PZT. Therefore, the sound level attenuation is below $3 \mathrm{~dB}$.

Despite the better performance of PVDF in a wider band of frequencies, its $d_{33}$ coupling is 15 times lower than PZT-5H. This drawback can be overcome by implementing multilayer structures [1,17-20].

Three different transducers were tested: PVDF and PZT-5H transducers with a single layer of $110 \mu \mathrm{m}$ thicknesses and $1 \mathrm{~cm}$ of diameter and a PVDF transducer with four layers of $28 \mu \mathrm{m}$ thickness and $1 \mathrm{~cm}$ diameter. The transducer of $110 \mu \mathrm{m}$ thickness of PVDF has a resonance frequency of $10 \mathrm{MHz}$, the PZT-5H transducer of the same thickness has a resonance frequency of $20 \mathrm{MHz}$, and the

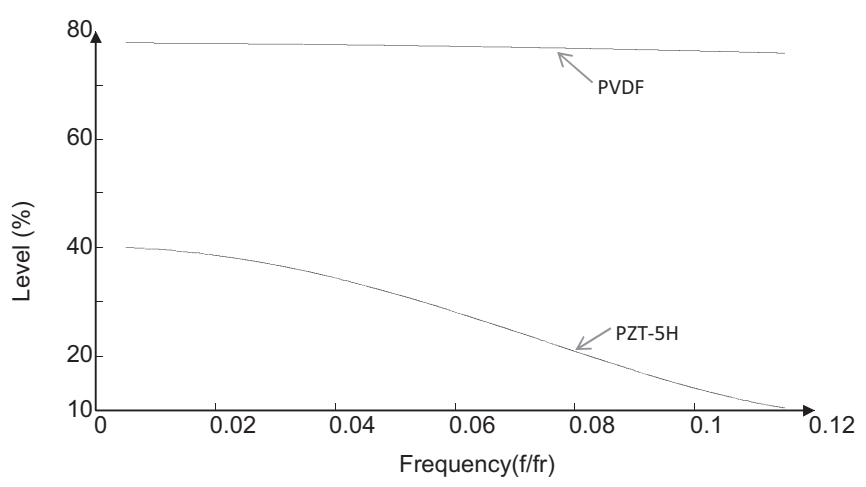

Fig. 6. PVDF and PZT-5H sound level response over 7 cycles below $0.11 \mathrm{fr}$.

transducer with four layers of PVDF has a resonance frequency of $10 \mathrm{MHz}$. So, if frequencies below $1 \mathrm{MHz}$ are used, the transmission coefficient ratios of the sound power level do not show significant flotation. The tests were performed at a frequency band from $100 \mathrm{kHz}$ to $2 \mathrm{MHz}$.

\section{Transducer design and fabrication}

The single layer transducers have the structure represented in Fig. 3, where the active element has $110 \mu \mathrm{m}$ of thickness and $1 \mathrm{~cm}$ of diameter. The multilayer one has the structure shown in Fig. 7, where the active element has four layers of $28 \mu \mathrm{m}$ of thickness and $1 \mathrm{~cm}$ of diameter. The electrodes connections, as shown in Fig. 7 , allow removing the parasite capacitance between the electrodes and the glue. By bonding reversed polarization layers, the electrodes between the glue have the same electrical potential, and

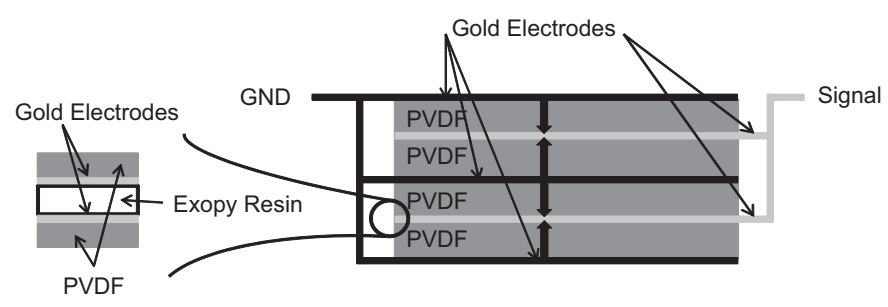

Fig. 7. 2D representation of the active element multilayer structure. 


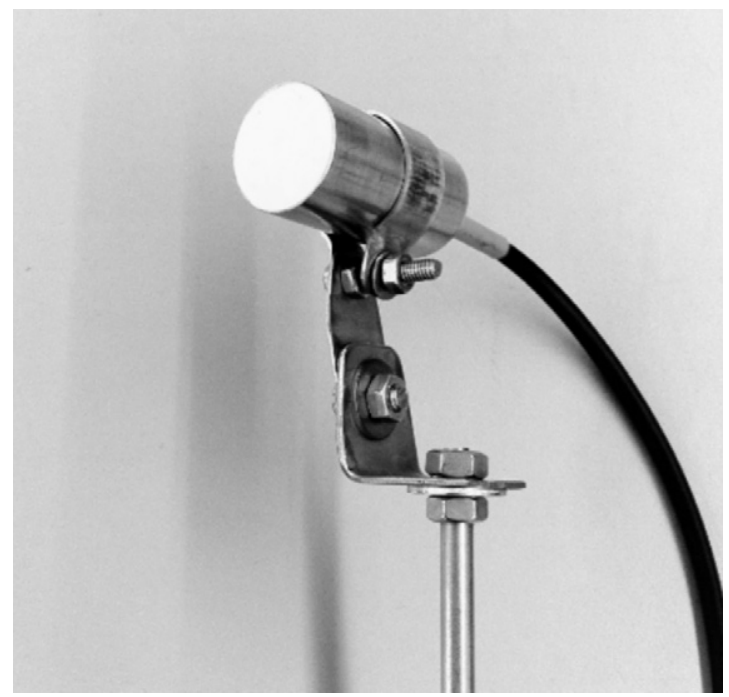

Fig. 8. Final transducer set up.

there is no current between them and consequently reduce the power consumption once the parasite capacitance was disabled.

The Gold electrodes with $10 \mathrm{~mm}$ circular shape were deposited by sputtering in both sides of the film, with a Polaron SC502 sputter coater. The four layers of the transducer were then glued with an epoxy resin Devcon 5 minute ${ }^{\odot}$, in a compression press. Finally, the active element was glued to a stainless steel mass and the active element outside surface was isolated with a thin silicone layer to protect it from water. Fig. 8 shows a picture of the final device.

\section{Simulation and experimental setup}

In order to compare the behavior of PZT-5H with PVDF single and multiple layers, the mechanical and electrical proprieties of each transducer were theoretically calculated; a computational simulation was performed and, finally, experiments in a water tank were conducted.

To a better understanding of the comparative performance between the single-layer PZT, four-layer PVDF and the single-layer PVDF (reference), the relative performance graphics between two transducers and the reference are obtained from the following equation:

$20 \log _{10}\left(\frac{p}{p_{\text {ref }}}\right)$

where $p$ is the measured transducer pressure wave and $p_{\text {ref }}$ is the reference transducer pressure wave.

\subsection{Simulations}

The transducers performance for the PZT-5H with PVDF single and multiple layers were previous simulated. The finite element platform used was the Comsol Multiphysics in a 2D symmetric plane with the models Piezo Strain Plane for the active element actuation and the model Pressure Acoustic for the pressure waves. The simulations setup is the same for all transducers, where only the active element is replaced. The outside boundaries of the medium were configured as match boundaries to work as absorbers, removing all the echoes. The selected mesh has particles with triangular shape and a size of $10 \%$ of the wavelength. With this mesh the results of all simulations have 10 samples for each wavelength regardless the frequency of simulation signal.
The simulations were performed with the following settings: fresh water as propagation medium, $20^{\circ} \mathrm{C}$ of temperature and sine wave excitation signal with $10 \mathrm{~V}$ of amplitude. The measurements were taken $3 \mathrm{~cm}$ away from the transducer in order to ensure that the measurement is not taking place inside the near field of the sound beam where there is some turbulence.

\subsection{Experimental}

The real trails were implemented with the same conditions as the simulations: fresh water as propagation medium, $20^{\circ} \mathrm{C}$ of temperature, $10 \mathrm{~V}$ sine wave excitation signal and a distance of $3 \mathrm{~cm}$. The experimental setup consisted in a water tank with $1.2 \mathrm{~m} \times 0.5 \mathrm{~m} \times 0.4 \mathrm{~m}$ (length, width, height). With these dimensions echoes occurs very easily and the hydrophone surface work as sound reflector. It was not suitable the elimination of echoes, but it was possible to ensure the same settings for the three transducers tested.

The ultrasonic receptor, used to register the pressure waves, was the Cetacean Research ${ }^{\mathrm{TM}}$ C304XR hydrophone, with an effective sensibility of $181 \mathrm{~dB}$, re $1 \mathrm{~V} / \mu \mathrm{Pa}$ and a usable range of $0.005-2000 \mathrm{kHz}$.

\section{Simulations and experimental tests}

Before the simulations and experimental tests were carried out, it was necessary to analyze the potential transducers behavior. An ideal system was considered using the equations referred above, where the active element has a homogeneous displacement. Table 3 shows the materials features and output response analysis of the three transducers (PZT-5H $110 \mu \mathrm{m}$, PVDF $110 \mu \mathrm{m}$ and PVDF $4 \times 28 \mu \mathrm{m})$.

It is important to notice that, by dividing the active element into layers without changing its final thickness, the maximum frequency does not change, but the equivalent excitation voltage, free displacement and maximum applied force are multiplied by the number of layers. If the active element thickness is not divided according the number of layers, the multi structure transducer was not able to achieve the same single layer maximum frequency, once the maximum applied force remains the same but the free displacement is multiplied by the number of layers. In the four-layer case, the expected performance increases $12.04 \mathrm{~dB}$, for PZT the expected increase in performance is $23.56 \mathrm{~dB}$.

\subsection{Simulations}

A transducer 2D model was designed and simulated for each topologies described in Section 4 "Transducer Design and Fabrication" and Section 5 "Simulation and Experimental Setup".

To better understand the difference of performance among the three topologies, Fig. 9 shows the simulated relative performance according equation Eq. (A.16) between single-layer PZT versus single-layer PVDF, four-layer PVDF versus single-layer PVDF and expected values, respectively.

Analyzing Fig. 9 it was possible to observe that the ratio of PZT versus PVDF performance is slightly below than the expected value and the four-layer PVDF versus single-layer PVDF performance match the expected values with the exception of values to frequencies above $1.6 \mathrm{MHz}$ where the graphic shows a performance growth. This growth results from the fact that multilayer structures with thinner layers will have different mechanical, morphological and structural characteristics [17-30]. 
Table 3

PZT-5H $110 \mu \mathrm{m}$, PVDF $110 \mu \mathrm{m}$ and PVDF $4 \times 28 \mu \mathrm{m}$ analysis considering homogeneous displacements.

\begin{tabular}{|c|c|c|c|}
\hline Feature & PZT $110 \mu \mathrm{m}$ & PVDF $110 \mu \mathrm{m}$ & PVDF $4 \times 28 \mu \mathrm{m}$ \\
\hline Thickness (m) & $1.10 \mathrm{E}-04$ & $1.10 \mathrm{E}-04$ & $2.80 \mathrm{E}-05$ \\
\hline Resonance frequency $(\mathrm{Hz})$ & $2.00 \mathrm{E}+07$ & $1.02 \mathrm{E}+07$ & $1.00 \mathrm{E}+07$ \\
\hline Sound speed $(\mathrm{m} / \mathrm{s})$ & $4.40 \mathrm{E}+03$ & $2.25 E+03$ & $2.25 E+03$ \\
\hline Density $\left(\mathrm{kg} / \mathrm{m}^{3}\right)$ & $7.50 \mathrm{E}+03$ & $1.47 \mathrm{E}+03$ & $1.47 \mathrm{E}+03$ \\
\hline$d_{33}(\mathrm{C} / \mathrm{N})$ & $5.12 \mathrm{E}-10$ & $3.40 \mathrm{E}-11$ & $3.40 \mathrm{E}-11$ \\
\hline$S_{33}^{E}(1 / \mathrm{Pa})$ & $2.07 \mathrm{E}-11$ & $4.72 \mathrm{E}-10$ & $4.72 \mathrm{E}-10$ \\
\hline Number of layers & 1 & 1 & 4 \\
\hline Electrical potential (V) & 10 & 10 & 10 \\
\hline Free displacement (m) & $5.12 \mathrm{E}-09$ & $3.40 \mathrm{E}-10$ & $1.36 \mathrm{E}-09$ \\
\hline Max applied force $(\mathrm{N})$ & $1.77 \mathrm{E}+02$ & $5.14 \mathrm{E}-01$ & $2.02 E+00$ \\
\hline Max frequency $(\mathrm{Hz})$ & $4.66 \mathrm{E}+07$ & $2.04 \mathrm{E}+06$ & $2.01 \mathrm{E}+06$ \\
\hline Test frequency $(\mathrm{Hz})$ & $1.00 \mathrm{E}+06$ & $1.00 \mathrm{E}+06$ & $1.00 \mathrm{E}+06$ \\
\hline Transmitted wave percentage at $1 \mathrm{MHz}(\%)$ & $32 \%$ & $76 \%$ & $78 \%$ \\
\hline Expected performance versus PVDF single layer (dB) & 21.92 & - & 12.19 \\
\hline
\end{tabular}

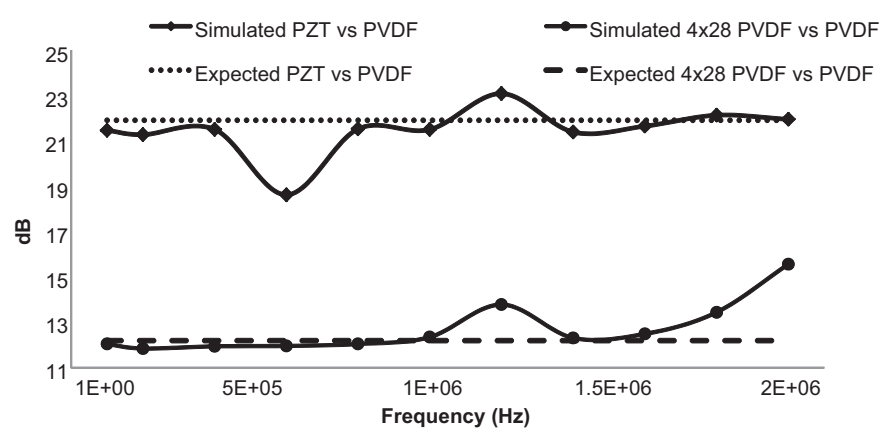

Fig. 9. Simulations performance improvement results of PZT versus single-layer PVDF, four-layers PVDF versus single-layer PVDF and expected values, respectively.

\subsection{Experimental}

Fig. 10 shows the experimental pressure wave measurements, where it is possible to observe the behavior of the three transducers over frequency.

In Fig. 10 it is possible to observe two peaks in the three transducer measurements: one at $400 \mathrm{kHz}$ and the other at $800 \mathrm{kHz}$. These peaks are due to a set of features of the experimental setup. By considering Eq. (A.14) it is possible to understand that, at low frequencies, the emitter presents a pattern with a wide divergence angle and, to these frequencies, the signal is emitted to the sides, where it is rebound back to the receiver. At higher frequencies the signal has a more directional pattern which prevents side echoes. Considering all these facts together, the peaks are the result of the tank resonance signal. Another fact that helps to conclude that these peaks do not result from the transducer but from the experimental setup is the graphics shown in Fig. 12, where it is possible

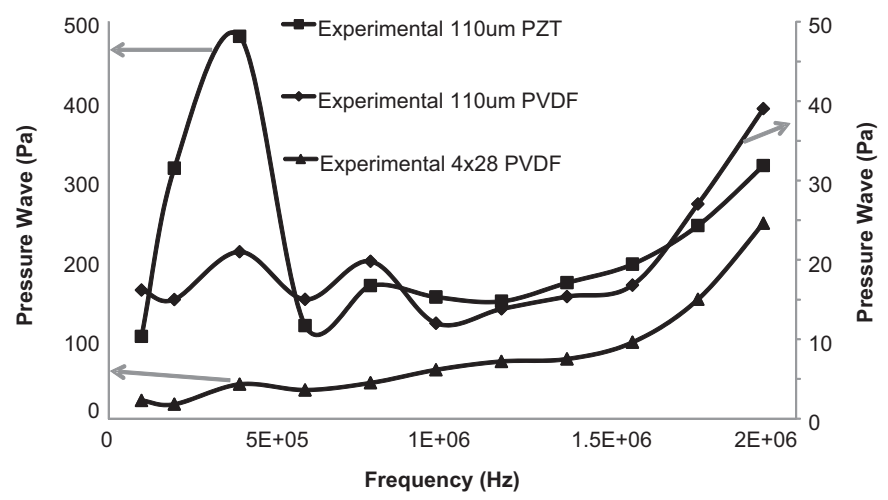

Fig. 10. Pressure wave responses over frequency of single layer PZT (left axis), four layer PVDF (left axis) and single layer PVDF (right axis).

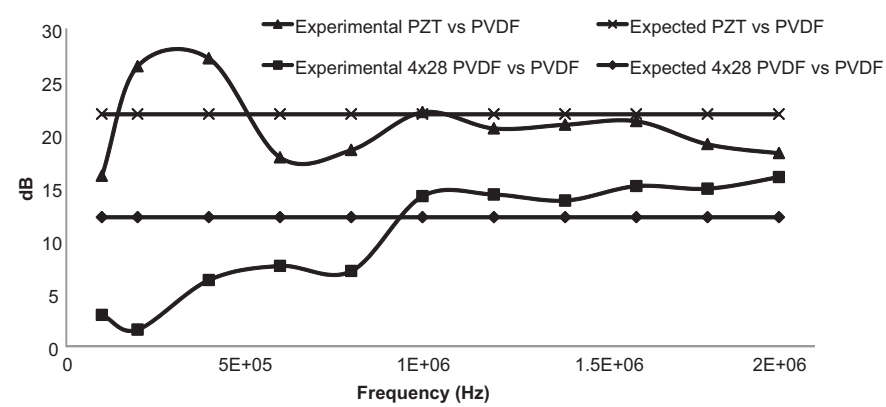

Fig. 11. Performance results of four-layers PVDF versus single-layer.

to observe that the electric current consumption over frequencies does not show any type of flotation, typical of the resonance harmonics and anti-resonance points.

Fig. 11 shows the relative performance results from the tank experiments according to Eq. (A.16) among single-layer PZT versus single-layer PVDF, four-layer PVDF versus single-layer PVDF and expected values, respectively.

In the PZT results shown in Fig. 11, not considering the initial peak, the results are very similar to the simulations results and shown an average performance of $20 \mathrm{~dB}$ versus single layer PVDF. However, in the four-layer PVDF case, the results are more interesting and can be divided in two parts. In the first part, between $100 \mathrm{kHz}$ and $1 \mathrm{MHz}$, the results are far below of the expected values, although show a continuous growth beginning at $100 \mathrm{kHz}$ with $3 \mathrm{~dB}$ and reaching the $14.2 \mathrm{~dB}$ at $1 \mathrm{MHz}$. In the second part, between $1 \mathrm{MHz}$ and $2 \mathrm{MHz}$, the growth rate slows down and, at $2 \mathrm{MHz}$, shows a performance improvement of $16 \mathrm{~dB}$.

It is possible to increase the transducer performance using multilayers, but this optimization has increases energy consumption as shown in Fig. 12.

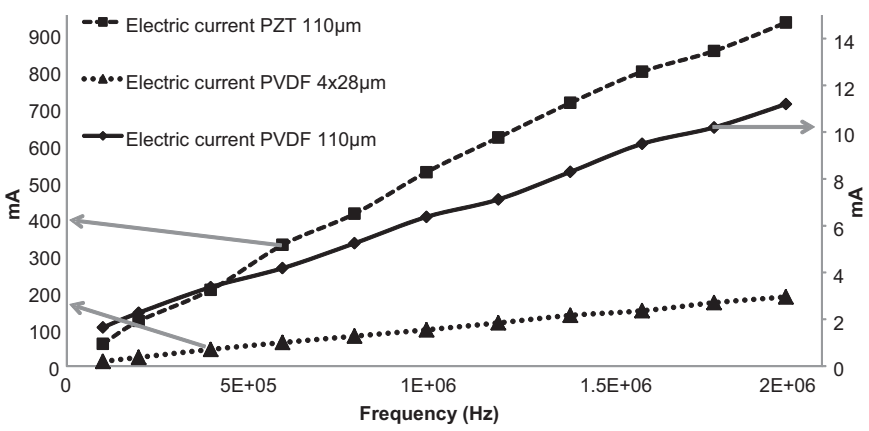

Fig. 12. Electric current consumption over frequency to PZT $110 \mu \mathrm{m}$ (left scale) PVDF $4 \times 28 \mu \mathrm{m}$ (left scale) and PVDF $110 \mu \mathrm{m}$ (right scale). 


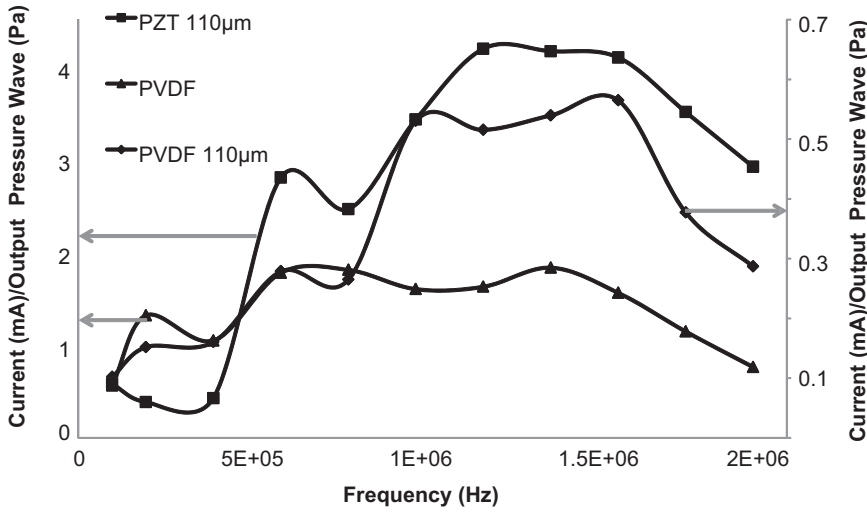

Fig. 13. Electric current consumption per Pascal of single layer PZT (left axis), fourlayer PVDF (left axis) and single layer PVDF (right axis).

The results shown in Fig. 12 allow visualizing the electric current consumption and they are according to theoretical analysis where the electrical current is directly proportional to the frequency. The PZT electric current consumption is 87 times higher than singlelayer PVDF duo to the high values of PZT relative dielectric constant. However the four-layer PVDF only shows 16.7 times more electric current consumption than single-layer PVDF and this occurs due to two factors: there is 4 times more effective area in the four-layer transducer and the distance between electrodes it is 4 times lower. It is possible to reduce the electric current consumption by increasing the film thickness but it is important to consider the effect that this change will introduce in the transducer performance. According to Eq. (A.8), the resultant force decline with increasing thickness. Another way to reduce the electric current consumption is to reduce the transducer area but this change will affect the sound beam wide and divergence angle.

Fig. 13 shows the electric current consumption per Pascal of the three topologies single-layer PZT-5H, four-layer PVDF and singlelayer PVDF.

Fig. 13 helps to understand the efficiency level of each transducer. Despite the low outcome of single-layer, PVDF shows to be the most efficient concerning electric current consumption per Pascal, followed by the four-layer PVDF and the less efficient the single-layer PZT. By ignoring the peaks related to experimental constrains, the average electric current consumption of the tree topologies are $3.5 \mathrm{~mA} / \mathrm{Pa}$ for the single-layer PZT, $1 \mathrm{~mA} / \mathrm{Pa}$ for the four-layer PVDF and $0.3 \mathrm{~mA} / \mathrm{Pa}$ for the single-layer PVDF.

\section{Conclusions}

An optimization study for ultrasonic transducers for underwater communications was performed. Three different designs with piston topology were compared, namely PZT-5H single-layer, PVDF single-layer and PVDF four-layer.

The study includes calculations, finite element simulation and experimental tests.

From the three studied topologies studied, the PZT transducer achieves higher output sound pressure, but the four-layer PVDF demonstrates to be the best for this kind of applications, due to its higher bandwidth and lower power consumption.

In this study, the single-layer PZT output sound pressure shows to be in average $7 \mathrm{~dB}$ higher than four-layer PVDF, but also shows an average power consumption of 3.5 times higher.

\section{Acknowledgements}

The authors thank the FCT for financial support under grant PTDC/CTM/69316/2006. M. S. Martins thanks the FCT for the grant
SFRH/BD/60713/2009. V. Correia thanks the FCT for the grant SFRH/BD/48708/2008.

\section{References}

[1] Donald J. Leo, Engineering Analysis of Smart Material Systems, John Wiley \& Sons, Inc., 2007, ISBN 978-0-471-68477-0.

[2] Charles H. Sherman, John L. Butler, Transducers and Arrays for Underwater Sound, Springer Science Business Media, LLC, 2007, ISBN-10: 0-387-32940-4.

[3] J.J. Leonard, B.A. Moran, I.J. Cox, M.L. Miller, Underwater sonar data fusion using an efficient multiple hypothesis algorithm, in: Proceedings of 1995 IEEE International Conference on Robotics and Automation, vol. 3, 21-27 May 1995, 1995, pp. 2995-3002, http://dx.doi.org/10.1109/ROBOT.1995.525709.

[4] P.G. Auran, O. Silven, Underwater Sonar Range Sensing and 3d Image Formation", Control Engineering Practice 4 (3) (1996) 393-400, Elsevier Science.

[5] David F. Waechter, S. Eswar Prasad, Richard G. Blacow and Bin Yan, Internally biased PZT materials for high-power sonar transducers, 11th CF/DRDC International Meeting on Naval Applications of Materials Technology.

[6] D.A. Hughes, J.M. Girkin, S. Poland, C. Longbottom, T.W. Button, J. Elgoyhen, H. Hughes, C. Meggs, S. Cochran, Investigation of dental samples using a $35 \mathrm{MHz}$ focused ultrasound piezocomposite transducer, Ultrasonics 49 (February (2)) (2009) 212-218, Elsevier Science ISSN 0041-624X.

[7] M. Stojanovic, On the relationship between capacity and distance in an underwater acoustic communication channel, ACM SIGMOBILE Mobile Computing and Communications Review (MC2R) 11 (October (4)) (2007) 34-43.

[8] I.A. Sehgal, J. Schonwalder, Aquatools: An Underwater Acoustic Networking Simulation Toolkit, IEEE, Oceans, Sydney, Australia May, 2010.

[9] M. Stojanovic, J. Presig, Underwater acoustic communication channels: propagation models and statistical characterization, IEEE Communications Magazine (January) (2009) 84-89.

[10] M. Stojanovic, Underwater acoustic communications: design considerations on the physical layer, in: IEEE/IFIP Fifth Annual Conference on Wireless on demand Network Systems and Services (WONS 2008), January, 2008.

[11] E.M. Sozer, M. Stojanovic, J.G. Proakis, Underwater acoustic networks, IEEE Journal of Oceanic Engineering 25 (1) (2000) 72-83, http://dx.doi.org/10.1109/48.820738.

[12] J. Preisig, Acoustic propagation considerations for underwater acoustic communications network development, Underwater Networks (WUWNet'06) (September) (2006) 1-5.

[13] I.A. Sehgal, J. Schonwalder, Variability of Available Capacity due to the Effects of Depth and Temperature in the Underwater Acoustic Communication Channel, OCEANS 2009-EUROPE, May, 2009, pp. 1-6.

[14] R.E. Francois, G.R. Garrison, Sound absorption based on ocean measurements: Part I: Pure water and magnesium sulfate contributions, Journal of the Acoustical Society of America 72 (3) (1982) 896-907.

[15] Malcolm J.W. Pavey, Ultrasonic Techniques for Fluids Characterization, Academic Press, 1997, ISBN-13: 978-0-12-563730-5 ISBN-IO: 0-12-563730-6.

[16] M.A. Ainslie, J.G. McColm, A simplified formula for viscous and chemical absorption in sea water, Journal of the Acoustical Society of America 103 (3) (1998) 1671-1672.

[17] Thomas R. Shrout, Innovations in piezoelectric materials for ultrasound transducers, applications of ferroelectrics, ISAF, in: 17th IEEE International Symposium, 2008.

[18] A. Abrar, S. Cochran, Multilayer piezocomposite structures with piezoceramic volume fractions determined by mathematical optimization, Ultrasonics 42 (April (1-9)) (2004) 259-265, Elsevier Science ISSN 0041-624X.

[19] Qian Zhang, Peter A. Lewin, Philip E. Bloomfield, PVDF transducers-a performance comparison of single-layer and multilayer structures, IEEE Transactions on Ultrasonics, Ferroelectrics and Frequency Control 44 (September (5)) (1997).

[20] A. Raúl, Reyes-Villagrana, Gerardo Gutiérrez-Juárez, Rumen Ivanov Tsonchev, Characterization of simulated mechanical-electrical properties of PVDF and PZT piezoelectric material for use in the pulsed optoacoustic spectroscopy, International Journal of Pure and Applied Sciences and Technology (2011) 26-45.

[21] L.W. Schmerr, A. Lopez-Sanchez, R. Huang, Complete ultrasonic transducer characterization and its use for models and measurements, Ultrasonics 44 (December (Suppl. 22)) (2006) e753-e757, Elsevier Science ISSN 0041-624X.

[22] Torsten Bove, Wanda Wolny, Erling Ringgaard, Annette Pedersen, New piezoceramic PZT-PNN material for medical diagnostics applications, Journal of the European Ceramic Society 21 (10-11) (2001) 1469-1472.

[23] S. Saitoh, T. Kobayashi, K. Harada, S. Shimanuki, Y. Yamashita, A $20 \mathrm{MHz}$ single-element ultrasonic probe using $0.91 \mathrm{~Pb}(\mathrm{Zn} 1 / 3 \mathrm{Nb} 2 / 3) \mathrm{O} 3-0.09 \mathrm{PbTiO} 3 \mathrm{sin}-$ gle crystal, IEEE Transactions on Ultrasonics, Ferroelectrics and Frequency Control 45 (1998) 1071-1076.

[24] T. Ritter, K.K. Shung, X. Geng, P.D. Lopath, S.E. Park, T.R. Shrout, Single crystal PZN- Tpolymer composites for ultrasound transducer applications, IEEE Transactions on Ultrasonics, Ferroelectrics and Frequency Control 47 (2000) 792-800.

[25] S. Saitoh, T. Takeuchi, T. Kobayashi, K. Harada, S. Shimanuki, Y. Yamashita, Fortychannel phased array ultrasonic probe using $0.91 \mathrm{~Pb}(\mathrm{Zn} 1 / 3 \mathrm{Nb} 2 / 3) \mathrm{O} 3-$ $0.09 \mathrm{PbTiO} 3$ single crystal, IEEE Transactions on Ultrasonics, Ferroelectrics and Frequency Control 46 (1999) 152-157.

[26] Linxiang Wang, M. Willatzen, Modelling of nonlinear dynamics for reciprocal multi-layer piezoceramic transducer systems, Applied Mathematical Modelling 33 (May (5)) (2009) 2263-2273, Elsevier Science ISSN 0307-904X. 
[27] F. Levassort, L.P. Tran-Huu-Hue, G. Feuillard, M. Lethiecq, Characterisation of $\mathrm{P}(\mathrm{VDFTTFE})$ material taking into account dielectric relaxation: application to modelling of high frequency transducers, Ultrasonics 36 (1-5) (1998) 41-45.

[28] K.A. Snook, J.-Z. Zhao, C.H.F. Alves, J.M. Cannata, W.-H. Chen, R.J. Meyer, T.A. Ritter, K.K. Shung, Design, fabrication, and evaluation of high frequency, singleelement transducers incorporating different materials, IEEE Transactions on Ultrasonics, Ferroelectrics and Frequency Control 49 (2002) 169-176.

[29] Yongrae Roh, Vasundara V. Varadan, Vijay K. Varadan, Characterization of all the elastic, dielectric, and piezoelectric constants of uniaxially oriented poled PVDF films, IEEE Transactions on Ultrasonics, Ferroelectrics and Frequency Control 49 (June (6)) (2002).

[30] P.E. Bloomfield, Dielectric and piezoelectric properties of stacked and plated PVDF, $\mathrm{P}(\mathrm{VDF} / \mathrm{TeFe}), \mathrm{P}(\mathrm{VDF} / \mathrm{TrFE})$ and ceramic/rubber composite thick films, in: Applications of Ferroelectrics, 1994.ISAF '94, Proceedings of the Ninth IEEE International Symposium, 7 Aug, 1991, pp. 287-290.

\section{Biographies}

M. Martins received the graduation in Industrial Electronics Engineering and Master's degree in Industrial Electronics and Computer Engineering from University of Minho, Portugal in 2006 and 2008 respectively. Since 2011 he has been at the Technology Department of the Polytechnic Institute of Cávado and Ave, Portugal, where he is assistant professor. Currently he is a research and Ph.D. student at The University of Minho, where his work is focused in the area of underwater wireless communications, piezoelectric materials and ultrasonic.

V. Correia received the graduation in Industrial Electronics Engineering and Master's degree in Industrial Electronics and Computer Engineering from University of Minho, Guimarães, Portugal in 2006 and 2008 respectively. Actually he is a research in the area of nano-sensors, nano-actuators and wireless communications in
Electroactive Smart Materials group, from University of Minho. The current project that is developing is "smart materials for electronic textiles and biomedical applications".

José Cabral received the Diploma and M.Sc. degrees in Electrical Engineering and Computers from the University of Porto, Portugal, in 1988 and 1995, respectively. In 2005 he received the Ph.D. degree in Industrial Electronics Engineering, at University of Minho. Between 1988 and 1996 was research assistant at INESC (Computer and Systems Engineering Institute), where he was involved in R\&D Projects on the areas of high-speed communication networks and digital audio funded by European R\&D programmes. At present he is an assistant professor at the Industrial Electronics Department of University of Minho. His current research interests are: Underwater Communications Systems and Low Bit Rate Traffic Aggregation in Control Applications.

S. Lanceros-Mendez graduated in physics at the University of the Basque Country Leioa, Spain, in 1991. He obtained his Ph.D. degree in 1996 at the Institute of Physics of the Julius-Maximilians-Universität Würzburg, Germany. He was Research Scholar at Montana State University, Bozeman, MT, from 1996 to 1998 and visiting scientist at the A.F. Ioffe Physico-Technical Institute, St. Petersburg, Russia (1995), Pennsylvania State University, USA (2007) and University of Potsdam (2008). Since September 1998 he has been at the Physics Department of the University of Minho, Portugal where he is associate professor. His work is focused in the area of electroactive smart materials and its applications as sensors and actuators with more than 150 research papers and several patents.

J.G. Rocha received the B.Sc., M.Sc., and Ph.D. degrees in industrial electronics engineering from the University of Minho, Guimarães, Portugal, in 1995, 1999, and 2004 respectively. Since 2004 , he has been an assistant professor with the Department of Industrial Electronics, University of Minho, and a researcher with the Algoritmi Research Center, where he is involved in research on sensors and sensor interfaces. He was a visitor researcher at the Delft University of Technology, The Netherlands in 2001-2002. 